EPiC Series in Engineering
Volume 2, 2018, Pages 82-93
SUMO 2018- Simulating Autonomous
and Intermodal Transport Systems

\title{
Assessment of ACC and CACC systems using SUMO
}

\author{
Kallirroi N. Porfyri ${ }^{1}$, Evangelos Mintsis ${ }^{1 *}$ and Evangelos Mitsakis ${ }^{1}$ \\ ${ }^{1}$ Hellenic Institute of Transport, Centre for Research and Technology Hellas, Thessaloniki, Greece \\ kporfyriecerth.gr, vmintsisecerth.gr, emitecerth.gr
}

\begin{abstract}
Emerging developments in the field of automotive technologies, such as Adaptive Cruise Control (ACC) and Cooperative Adaptive Cruise Control (CACC) systems, are expected to enhance traffic efficiency and safety on highways and urban roads. For this reason, substantial effort has been made by researchers to model and simulate these automation systems over the last few years. This study aims to integrate a recently developed car-following model for ACC and CACC equipped vehicles in the microscopic traffic simulation tool SUMO; the implemented ACC/CACC simulation models originate from empirical ones, ensuring the collision-free property in the full-speed-range operation. Simulation experiments for different penetration rates of cooperative automated vehicles, desired time-gap settings and network topologies are conducted to test the validity of the proposed approach and to assess the impact of ACC and CACC equipped vehicles on traffic flow characteristics.
\end{abstract}

Keywords: connected and automated vehicle (CAV), Adaptive Cruise Control (ACC), Cooperative Adaptive Cruise Control (CACC), microscopic simulation

\section{Introduction}

In the last few decades, new technologies in the field of Advanced Driver Assistance Systems (ADAS) have been designed and deployed (at an increasing rate) to improve road safety and driving comfort, and increase capacity (Blythe and Curtis, 2004). Examples of such ADAS include Adaptive Cruise Control (ACC) and its more sophisticated variant Cooperative Adaptive Cruise Control (CACC) systems. These systems have recently received much interest by the automobile industry and various research institutions around the world. However, in order to ensure that such emerging systems will be developed and integrated in ways that further improve, rather than deteriorate the prevailing traffic flow conditions, it is important to gain insight in the impacts of such systems on the traffic dynamics (van Arem et al., 2006). Therefore, modelling and simulation of these new technologies is required, inter alia, for the design and testing of efficient control strategies in the future.

*Corresponding Name: Evangelos Mintsis. Tel.: +30-2310-498483

E-mail address: vmintsis@certh.gr

E. Wießner, L. Lücken, R. Hilbrich, Y.-P. Flötteröd, J. Erdmann, L. Bieker-Walz and M. Behrisch (eds.), SUMO2018 (EPiC Series in Engineering, vol. 2), pp. 82-93 
ACC systems that are currently available on the market enable automatic following of a preceding vehicle by controlling the throttle and/or the brake actuators of the ACC vehicle. Specifically, using range sensors, such as radar, lidar and video camera, an ACC system is able to measure the distance and the relative velocity with respect to a preceding vehicle. If the ACC sensors detect a slower preceding vehicle, ACC automatically adjusts the speed of the ACC equipped vehicle so as to maintain a desired space headway (gap-control mode). In the absence of a preceding vehicle, the ACC vehicle operates under the speed-control mode, maintaining the user pre-set desired speed. As an extension to ACC functionality, CACC systems are designed to exploit information provided by vehicle-to-vehicle (V2V) and/or vehicle-to-infrastructure (V2I) communication via wireless technology or ad-hoc networks. Thus, such enhanced systems offer high potential to further improve traffic safety and optimize traffic flow at road networks, since the CACC equipped vehicles can follow their predecessors with higher accuracy, faster response to changes, and shorter time gaps (Shladover et al., 2012).

Many studies address the impacts of ACC and CACC vehicles on traffic flow dynamics with the use of traffic simulation, since large-scale field tests are not currently feasible. However, some investigations predict a positive effect of both automated systems (Hasebe et al., 2003; Davis, 2004; van Arem et al., 2006; Treiber and Helbing, 2009; Naus et al.,2010;), whereas others are more conservative on the stabilization results of ACC systems (Marsden et al., 2001; Milanés and Shladover, 2014).

In (Liang and Peng, 1999) the authors suggested a two-level ACC synthesis method based on optimal control theory; the upper level calculates the desired acceleration rate depending on vehicle range (inter-vehicle distance in terms to its predecessor) and range rate (difference in the corresponding speeds) measurements, whereas the lower (servo) level deals with the conversion of the higher level acceleration command, into brake or throttle commands, accurately. Given that the control signal optimizes the range and range errors rate of all vehicles in the string, string stability is guaranteed.

In (VanderWerf et al., 2001) a set of mathematical models that incorporate ACC and CACC functionality was developed, aiming to predict the effects of the ACC and CACC vehicles on overall traffic flow dynamics and safety. A year later these models were used to investigate the effects of different vehicle types (manually driven vehicles, ACC vehicles, and CACC vehicles) on traffic flow capacity, for different market penetration rates (VanderWerf et al., 2002). This study showed that conventional ACC systems are unlikely to have significant positive or negative effects on traffic flow, while on the other hand, CACC functionalities could significantly increase the capacity per lane when using time gaps as short as 0.5 seconds.

In (Kesting et al., 2008) the Intelligent Driver Model (IDM) introduced by (Treiber et al., 2000) was used as a reference for incorporating ACC behaviour in traffic flow simulation. According to the simulation results, traffic congestion was eliminated with a low ACC market penetration (25\%) while significant improvements in travel times were produced for much lower penetration rates (5\%). This approach has been enhanced in (Kesting et al., 2010) by extending the IDM with a new constantacceleration heuristic (CAH) in order to avoid abrupt braking responses in case of cut-in manoeuvres; the IDM model was further modified in (Treiber and Kesting, 2013) for situations where the actual operating speed exceeds or is very close to the desired one. Experimental results stated that even at low penetration rates of ACC equipped vehicles (under 50\%) the dynamic road capacity is essentially improved.

Car-following control algorithms for ACC and CACC equipped vehicles were presented in (Shladover et al., 2012) that were actually implemented on test vehicles for field experiments and are proprietary to Nissan. The authors propose two modes, the speed control and the gap control mode, in the developed ACC/CACC control algorithm; the speed control enables vehicles to maintain their speed close to the desired speed limit, while gap control aims to maintain the desired gap between the controlled vehicle and its preceding one. Simulation results demonstrated that the increase in the number of ACC vehicles is unlikely to increase the capacity of the freeway significantly; on the other hand, at a high market penetration of CACC vehicles the traffic capacity can potentially double. Based 
on this approach, in (Milanés and Shladover, 2014) a new control system was introduced and evaluated on four production passenger cars, equipped with CACC and dedicated short-range communication (DSRC) systems to exchange information. Real traffic scenarios, including cut-in and cut-out manoeuvres, were conducted to compare the new CACC controller with respect to the commercially available ACC system. The results revealed that CACC system can improve the response time of the following vehicle, thereby improving the string stability.

This present work integrates two car-following models reflecting ACC and CACC behaviour in the microscopic traffic simulator SUMO; it builds upon recent work from (Xiao et al., 2017), where ACC and CACC simulation models originating from a commercial ACC controller (Milanés and Shladover, 2014), were established to guarantee the full-speed range operation of equipped vehicles while considering the collision avoidance constraint. The rest of the paper is structured as follows: In Section 2 the empirical ACC/CACC car-following model is presented, along with the model specifications and modifications. In Section 3 a brief description of SUMO is included. In Section 4 two different simulation scenarios are conducted to qualitatively evaluate the performance of the ACC and CACC equipped vehicles, compared to manually driven ones. The main findings of the study are summarized in Section 5.

\section{Microscopic Modelling of ACC and CACC Systems}

In this section empirical car-following models that simulate ACC and CACC behaviour are presented. These ACC and CACC models were developed based on data collected during field tests that measured the actual responses of following vehicles (Milanés and Shladover, 2014); thus, V2V communication is inherently approximated by the proposed mathematical formulations reflecting the CACC behaviour of following vehicles. The selected ACC/CACC driving models are settled on ( Milanes et al., 2014; Milanés and Shladover, 2014, 2016; Xiao et al., 2017; Liu et al., 2018) wherein the developed control law in the ACC/CACC control algorithm is explicitly divided into three modes based on three different motion purposes: speed (or cruising) control, gap-closing control and gap control. More specifically, the speed control mode is designed for maintaining the pre-defined by the driver desired speed, the gap control mode aims to maintain a constant time gap between the controlled vehicle and its predecessor, while the gap-closing controller enables the smooth transition from speed control mode to gap control mode. In the text below, the basic definitions and equations for these three parts of the ACC/CACC control algorithm are presented.

\subsection{ACC Control Algorithm}

\section{Speed control mode}

For the ACC car-following model, the feedback control law in speed mode is activated when there are no preceding vehicles in the range covered by the sensors or preceding vehicles exist in a spacing larger of $120 \mathrm{~m}$ (Xiao et al., 2017; Liu et al., 2018). This mode aims to eliminate the deviation between the vehicle speed and the desired speed and is given as:

$$
\alpha_{i, k+1}=k_{1}\left(v_{d}-v_{i, k}\right), \quad k_{1}>0
$$

where $\alpha_{i, k+1}$ represents the acceleration recommended by speed control mode of the $i$-th consecutive (subject) vehicle for the next time step $k+1 ; v_{d}$ and $v_{i, k}$ indicate the desired cruising speed and the speed of the $i$-th vehicle at the current time step $k$, respectively; and $k_{1}$ is the control gain determining the rate of speed deviation for acceleration. Typical values for this gain range between $0.3-0.4 s^{-1}$ according to (Xiao et al., 2017), and $0.4 s^{-1}$ is selected in this study. 
Gap control mode

In the case of the gap control mode, the acceleration in the next time step $k+1$ is modelled as a second-order transfer function based on the gap and speed deviations with respect to the preceding vehicle, and is defined as:

$$
\alpha_{i, k+1}=k_{2} e_{i, k}+k_{3}\left(v_{i-1, k}-v_{i, k}\right), \quad k_{2}, k_{3}>0
$$

in which $e_{i, k}$ is the gap deviation of the $i$-th consecutive vehicle at the current time step $k$, and $v_{i-1, k}$ is the current speed of the preceding vehicle (index $i-1$ refers to the leader of vehicle $i$ ); $k_{2}$ and $k_{3}$ are the control gains on both the positioning and speed deviations, respectively. The proposed optimal values for the gains are $k_{2}=0.23 s^{-2}$ and $k_{3}=0.07 s^{-1}$ (Xiao et al., 2017). The gap control mode is activated when the gap and speed deviations are concurrently smaller than $0.2 \mathrm{~m}$ and $0.1 \mathrm{~m} / \mathrm{s}$ respectively (Xiao et al., 2017).

Moreover, in this study, and following from (Milanes et al., 2014; Milanés and Shladover, 2014, 2016), the gap deviation of the $i$-th consecutive vehicle $\left(e_{i, k}\right)$ is defined as

$$
e_{i, k}=x_{i-1, k}-x_{i, k}-t_{d} v_{i, k}
$$

According to Equation (3), the gap deviation is calculated by the current position of the preceding vehicle $x_{i-1, k}$, the current position of the subject vehicle $x_{i, k}$, the current speed of the subject vehicle $v_{i, k}$ and the desired time gap $t_{d}$ of the ACC controller.

Gap-closing control mode

At this point, it is important to clarify that although ACC car-following models by Milanés and Shladover (Milanés and Shladover, 2016) take into account the gap-closing controller, the ACC longitudinal vehicle response under this mode has not been modelled. To overcome this shortcoming, in (Xiao et al., 2017) the gap-closing controller was derived by tuning the parameters of the existing gap controller and this approach is adopted in the current study. In this case, the gap-closing control mode is triggered when the spacing to the preceding vehicle is smaller than $100 \mathrm{~m}$, and the control gains of Equation (2) are set as $k_{2}=0.04 \mathrm{~s}^{-2}$ and $k_{3}=0.8 \mathrm{~s}^{-1}$. If the spacing is between $100 \mathrm{~m}$ and $120 \mathrm{~m}$, the controlled vehicle retains the previous control strategy to provide hysteresis in the control loop and perform a smooth transfer between the two strategies (Xiao et al., 2017; Liu et al., 2018).

\subsection{CACC Control Algorithm}

\section{Speed control mode}

The speed controller for CACC vehicles is the same with the ACC ones since the additional information exchange (either V2V or V2I) does not influence the vehicle cruising mode. This control mode is activated when the time-gap is larger than $2 s$ (Xiao et al., 2017; Liu et al., 2018) and is given as:

$$
\alpha_{i, k+1}=k_{4}\left(v_{d}-v_{i, k}\right), \quad k_{4}>0
$$

where the control gain $k_{4}$ is equal to $0.4 s^{-1}$.

Gap control mode

For the CACC car-following model, the speed of the equipped vehicles in the next time step $k+1$ is represented by a first-order transfer function, according to: 


$$
v_{i, k+1}=v_{i, k}+k_{5} e_{i, k}+k_{6} \dot{e}_{i, k}, \quad k_{5}, k_{6}>0
$$

where $\dot{e}_{i, k}$ is the derivative of the gap deviation $\left(e_{i, k}\right)$, and is defined as:

$$
\dot{e}_{i, k}=v_{i-1, k}-v_{i, k}-t_{d} \alpha_{i, k} .
$$

with $t_{d}$ being the desired time gap of the CACC controller. The values of the control gains $k_{5}$ and $k_{6}$ of Equation (5) are set as $0.45 s^{-2}$ and $0.25 s^{-1}$, respectively (Xiao et al., 2017). The gap control mode for CACC vehicles is activated when the gap and speed deviations are concurrently smaller than $0.2 \mathrm{~m}$ and $0.1 \mathrm{~m} / \mathrm{s}$ respectively (Xiao et al., 2017).

\section{Gap-closing control mode}

The gap-closing control mode is triggered when the time-gap is less than $1.5 \mathrm{~s}$, and as in the case of ACC car-following models, was derived by tuning the parameters of the existing gap controller according to (Xiao et al., 2017). Thus, the computed optimal gains of Equation (5) are $k_{5}=0.01 \mathrm{~s}^{-2}$ and $k_{6}=1.6 s^{-1}$. If the time-gap is between $1.5 s$ and $2 s$, the controlled vehicle retains the previous control strategy to provide hysteresis, as in case of the ACC control algorithm ( Xiao et al., 2017; Liu et al., 2018).

\section{The SUMO Simulation Environment}

The latter ACC and CACC car-following models were incorporated into the microscopic traffic simulation tool SUMO to execute the corresponding simulated experiments for the assessment of the effects of ACC/CACC vehicles on different traffic situations. SUMO (Simulator of Urban MObility) is an open-source, high-portable, and fast in run-time simulator created by the German Aerospace Centre DLR in 2001, which enables users to develop and integrate new algorithms; despite its name, SUMO can also simulate highway traffic networks (Behrisch et al., 2011). The simulator also includes the TraCI (Traffic Control Interface) tool, which is a Python API offering users the ability to interact with the running simulation in order to control the vehicle parameters.

The default-car following model implemented in SUMO is a modified version of the Krauss model, introduced by Stefan Krauss (Krauß, 1998), which is a collision-free model (each time step the following vehicle adapts its speed according to the speed of the preceding one). The main parameters of the implemented Krauss model in SUMO are the acceleration ability of vehicles (in $\mathrm{m} / \mathrm{s}^{2}$ ), accel, the deceleration ability of vehicles (in $\mathrm{m} / \mathrm{s}^{2}$ ), decel, the driver's imperfection in holding the desired speed (between 0 and 1), sigma, the length of vehicles (in $m$ ), length, the maximum velocity of vehicles (in $m / s$ ), maxSpeed, the minimum desired net distance (gap) to the leading vehicle (in $m$ ), minGap, and the driver's desired time headway (in $s$ ), tau.

\section{Simulation Experiments}

\subsection{Freeway Stretch}

The first experiment examines the impacts of ACC and CACC equipped vehicles on traffic flow capacity (compared to manual vehicles), for different penetration rates and time-gap settings; the carfollowing logic of manually driven vehicles is emulated based on the aforementioned Krauss model (default SUMO car-following model). Specifically, the simulated road is a single-lane freeway stretch 
without any on-ramps and off-ramps; the stretch is $6.5 \mathrm{~km}$ long, with a speed limit of $100 \mathrm{~km} / \mathrm{h}$; the total simulated time is $1 \mathrm{~h}$ and the simulation step is set equal to $0.1 \mathrm{~s}$. ACC and CACC vehicles have the same average maximum acceleration and deceleration values, bounded within $2 \mathrm{~m} / \mathrm{s}^{2}$; the same applies for manually driven vehicles. Moreover, using a speed deviation, provided by SUMO through the attributes speedFactor and speedDev, these values are randomly selected for each vehicle, so that vehicles do not exhibit identical behaviour (their parameter values follow the same distribution though). The reaction time for manual vehicles is equal to $0.7 s$, whereas for ACC and CACC equipped vehicles is $0.1 \mathrm{~s}$. At this point it important to highlight that in the case of CACC car-following model the leading vehicle type is also considered; namely, the ACC functionality is triggered if the preceding vehicle is not a CACC equipped one.

The penetration rate for both ACC and CACC equipped vehicles is $25 \%, 50 \%, 75 \%$ and $100 \%$ (this scheme resulted in a total of 9 different simulation scenarios). The desired time-gap for manual driving is $1.64 \mathrm{~s}$, while different penetration rates were selected for the desired time-gaps for ACC and CACC equipped vehicles (for all 8 scenarios encompassing ACC or CACC vehicles):

- ACC: $31.1 \%$ at $1.6 s, 18.5 \%$ at $1.4 s, 50.4 \%$ at $1.1 s$

- CACC: $31.1 \%$ at $1.1 s, 18.5 \%$ at $0.9 s, 50.4 \%$ at $0.7 s$

Figure 1 and 2 depict the effect of different penetration rates of ACC and CACC equipped vehicles on traffic flow capacity relative to manual vehicles ( $0 \%$ ACC and $0 \%$ CACC); it can be observed that the roadway capacity increases significantly when the penetration of both ACC (Figure 1) and CACC (Figure 2) equipped vehicles is increased. The increased traffic flow rate along the total length of the stretch for CACC traffic is more pronounced compared to ACC and manual traffic, mainly as a result of the smaller desired time-gap settings. Figure 3 illustrates the times series mean speed of the traffic flow for manual, ACC and CACC equipped vehicles; as it can be noticed the operation of the CACC controller smooths significantly velocity oscillations.

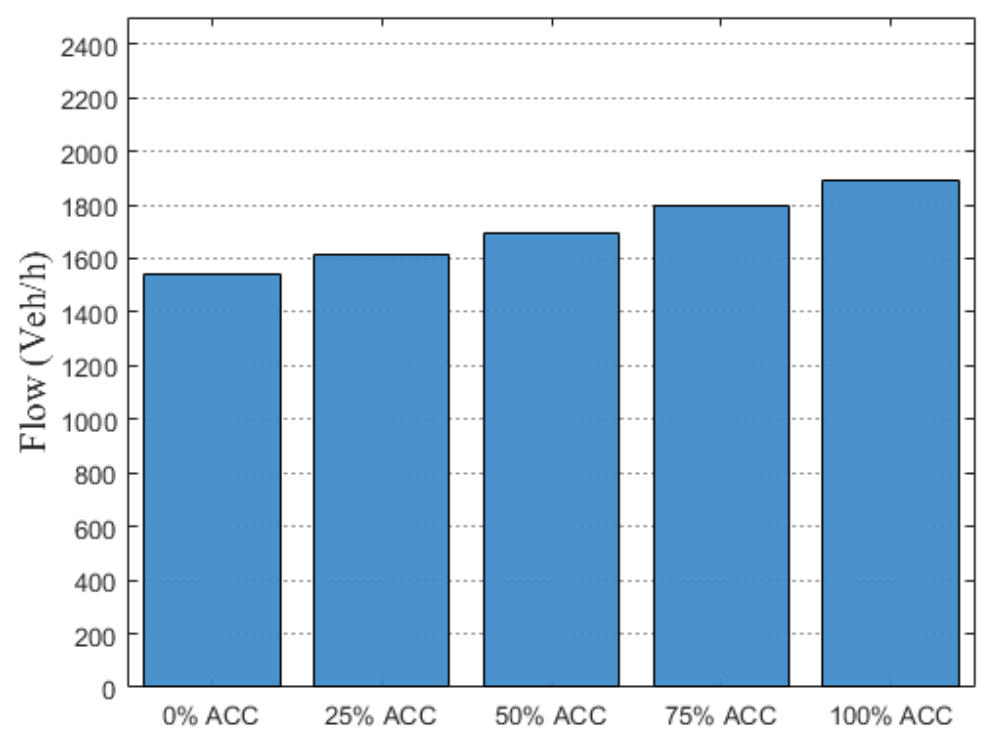

Figure 1: Flow for manually driven vehicles and different penetration rates of ACC equipped vehicles 


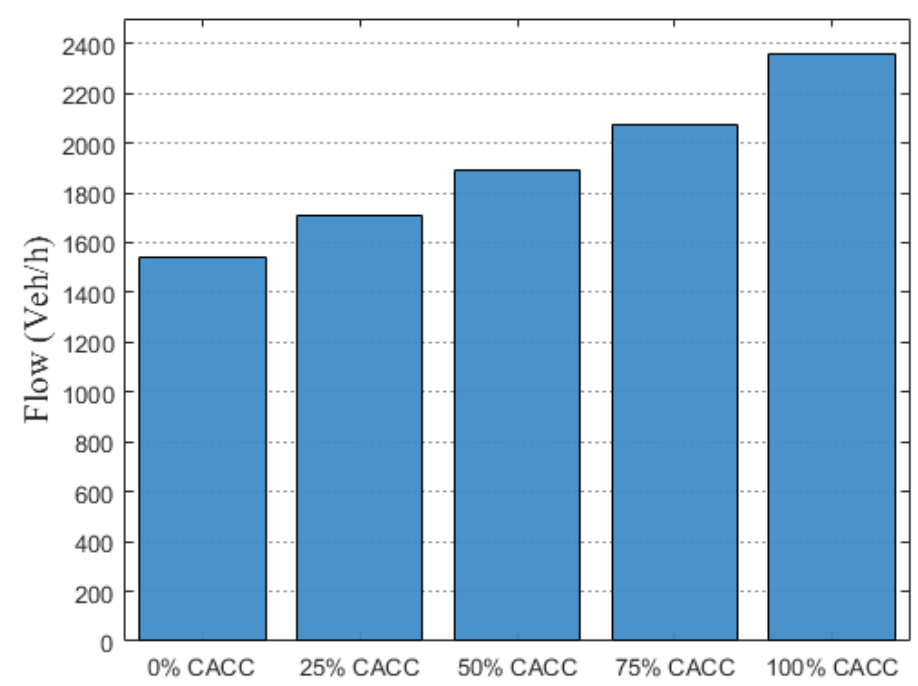

Figure 2: Flow for manually driven vehicles and different penetration rates of CACC equipped vehicles

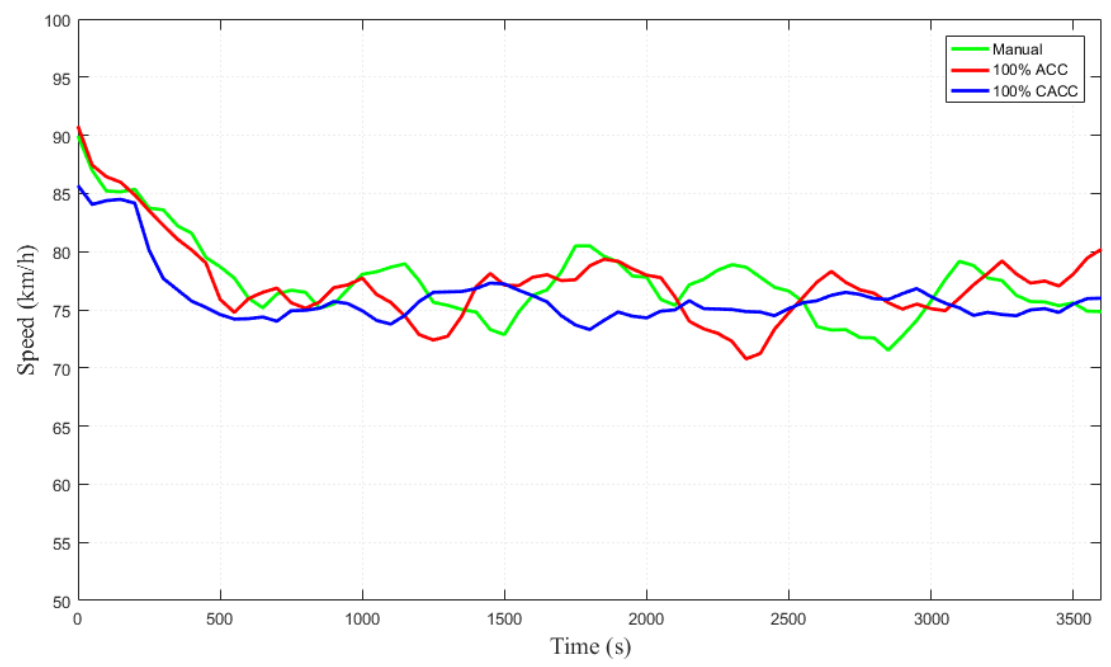

Figure 3: Average speed fluctuation for manual, ACC and CACC vehicles

\subsection{Ring-road}

In the second experiment, a single-lane ring-road of circumference of $L=4 \mathrm{~km}$ is considered to examine the stability property of ACC vehicles compared to manual ones. For the simulation of manually driven vehicles the IDM microscopic traffic flow model was used, which is also integrated in SUMO, as it can replicate with sufficient accuracy stop-and-go waves, unlike the Krauss model (Treiber and Kesting, 2013). A fixed size fleet of 200 identical vehicles was initially loaded in the network, resulting in an average density of $50 \mathrm{veh} / \mathrm{km}$. The flow data are recorded at intervals of $50 \mathrm{~s}$ from 80 detectors that were placed every $50 \mathrm{~m}$ in the ring-road. Thus, in order to examine the formation of traffic instabilities, a speed perturbation was introduced in the traffic flow, once the traffic equilibrium 
state was achieved, by decelerating a vehicle for $60 s$ while subsequently the vehicle accelerates again. Hence, the induced perturbation leads to a wave propagating upstream (Figure 4). Simulation runs are performed for $4000 \mathrm{~s}$, with a warm-up period of $2000 \mathrm{~s}$, while the simulation step is $0.1 \mathrm{~s}$. For both manual and ACC equipped vehicles a maximum acceleration $\alpha=1 \mathrm{~m} / \mathrm{s}^{2}$, and a maximum speed of $v=120 \mathrm{~km} / \mathrm{h}$ were used; the desired time-gap was set to $t_{d}=1.5 \mathrm{~s}$.

In Figure 4 it can be noticed that for $100 \%$ manual vehicles modelled with the IDM model the initial speed perturbation propagates upstream against the traffic flow producing multiple stop-and-go waves. The presence of ACC equipped vehicles (at a penetration rate of 50\%) can improve the stability of traffic flow reducing the formation of stop-and-go waves, as it is depicted in Figure 5. The phenomenon of multiple stop-and-go waves is less pronounced for the third case of $100 \%$ ACC equipped vehicles, as illustrated in Figure 6, since the perturbation fades-out quickly, having an observable smoothing effect on the oscillations.

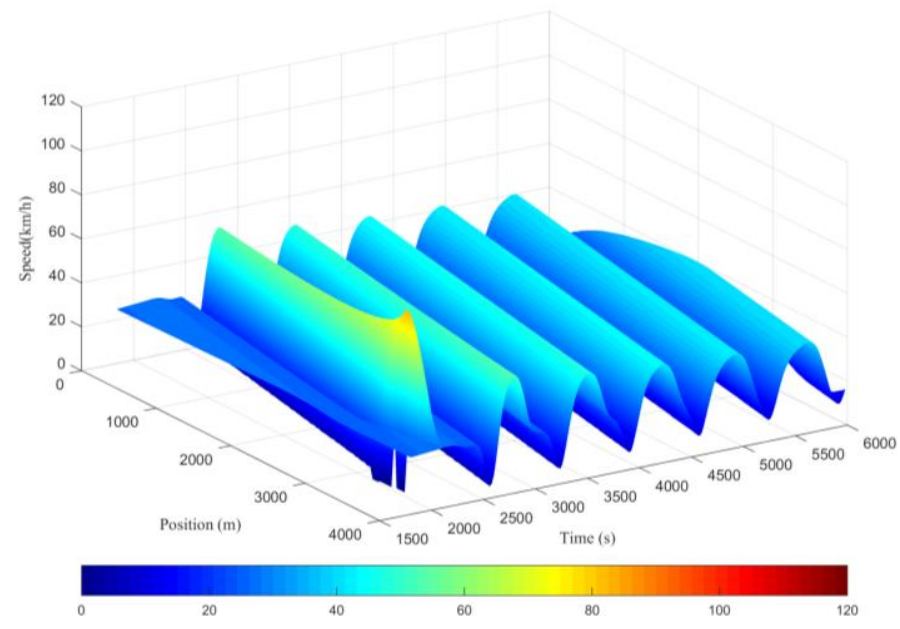

(a)

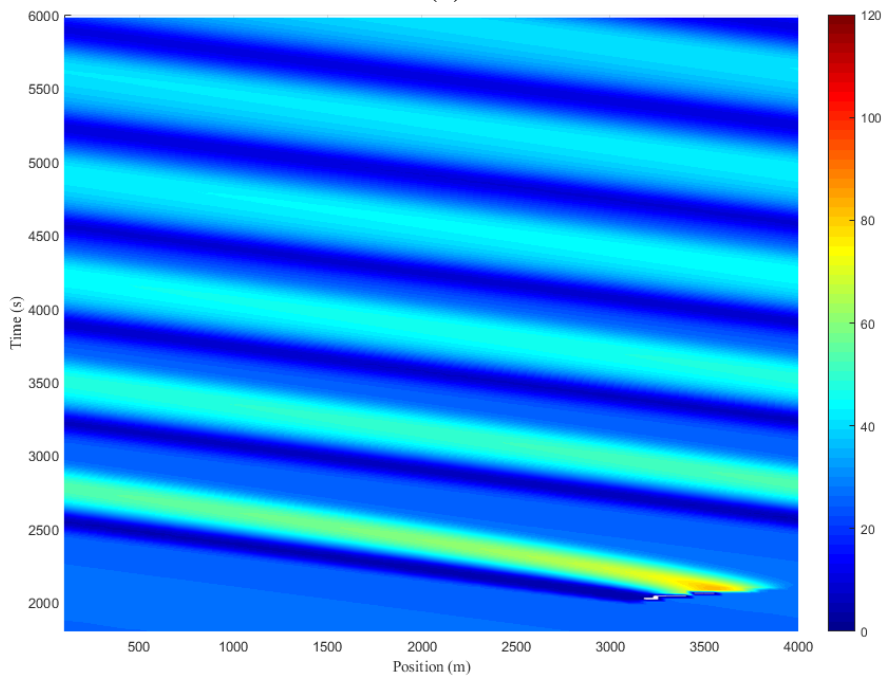

(b)

Figure 4: Space-time diagram of speed for $100 \%$ penetration rate of manually driven vehicles: (a) 3D plot, (b) 2D plot 


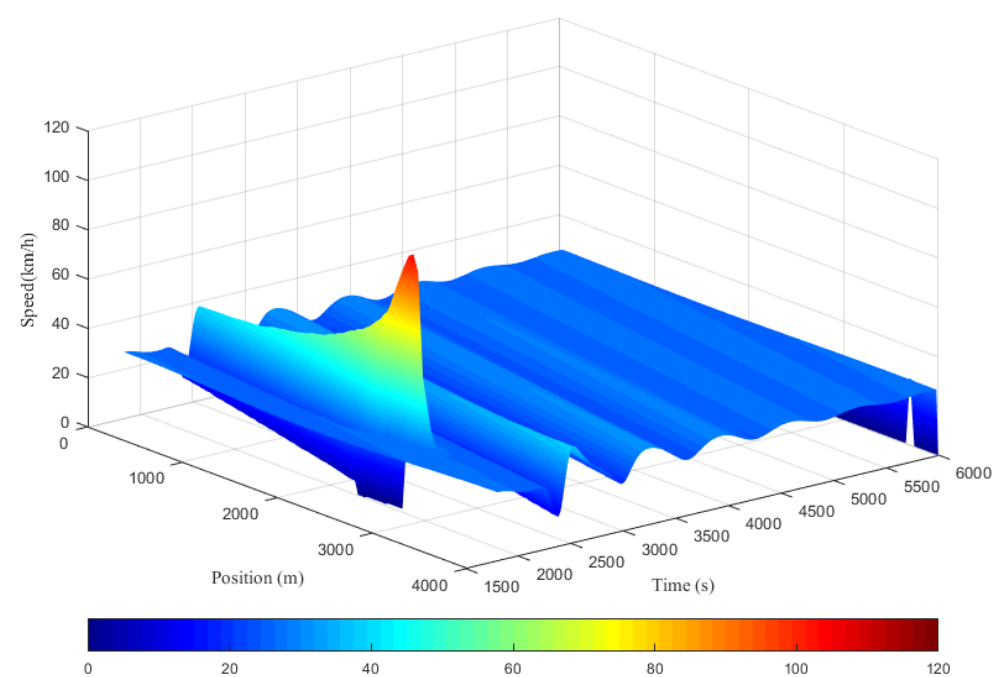

(a)

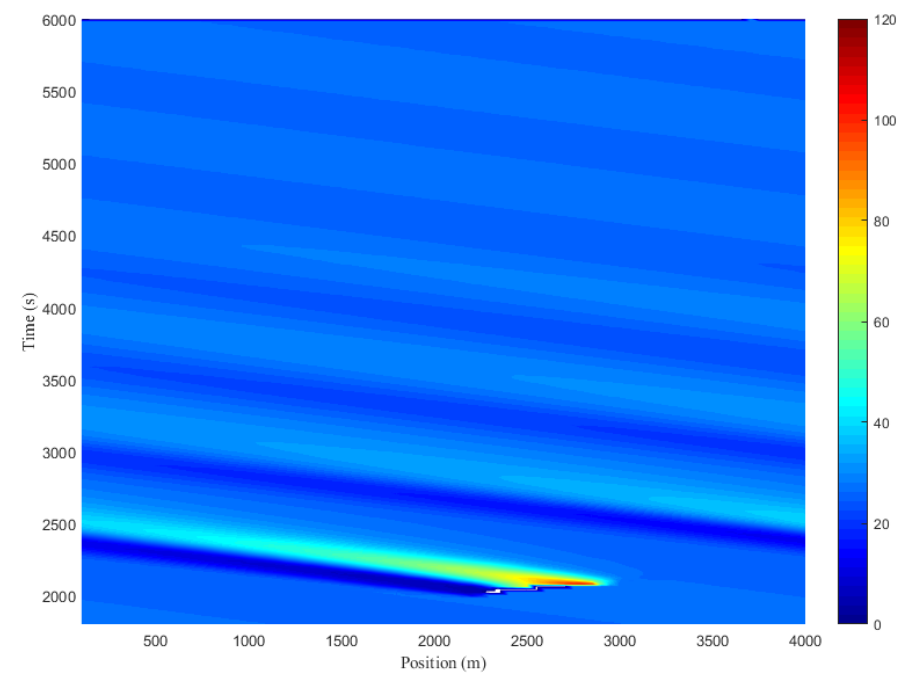

(b)

Figure 5: Space-time diagram of speed for 50\% penetration rate of manually driven vehicles and $50 \%$ penetration rate of ACC equipped vehicles: (a) 3D plot, (b) $2 \mathrm{D}$ plot 


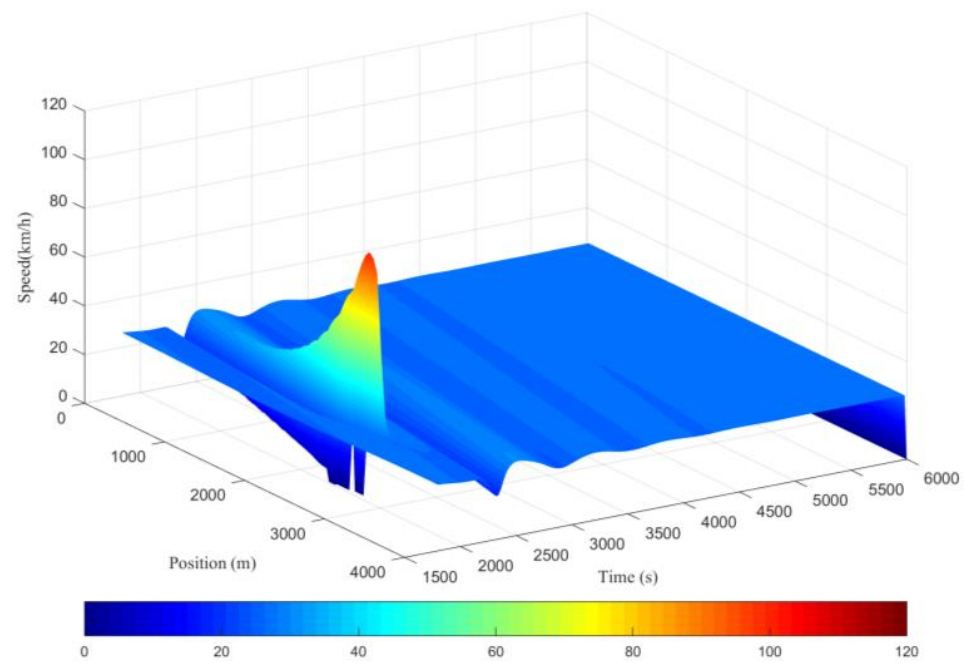

(a)

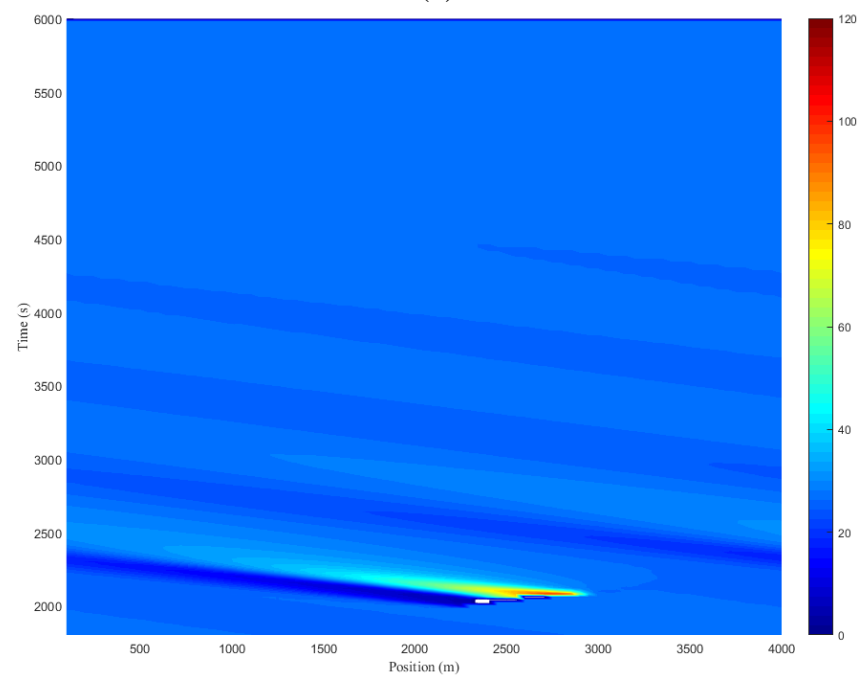

(b)

Figure 6: Space-time diagram of speed for $100 \%$ penetration rate of ACC equipped vehicles: (a) $3 \mathrm{D}$ plot, (b) $2 \mathrm{D}$ plot

\section{Conclusions}

This work describes the integration of ACC and CACC traffic dynamics in the microscopic traffic simulator SUMO and its use for investigating the impacts of ACC and CACC vehicles on traffic flow. Simulations were executed to investigate the effect of the ACC and CACC penetration rates to traffic dynamics. The results showed that both ACC and CACC systems have the potential to substantially improve the capacity even at low market penetration rates, since the desired time-gap settings are significantly shorter than that of conventional vehicles. Moreover, a second example was conducted, in 
a ring-road (with periodic boundary conditions) to verify stabilization effects on traffic flow when ACC equipped vehicles are considered. It was observed that ACC vehicles enhance the stability of traffic flow with respect to introduced perturbations compared to manual ones, since they are able to eliminate stop-and-go waves. Our ongoing work is investigating the contribution of CACC equipped vehicles to the stabilization of traffic flow. In addition, further validation of the developed microscopic approach incorporating the behaviour of ACC and CACC equipped vehicles is under development.

\section{Acknowledgements}

The research presented in the present paper has been conducted within the context of the TransAID (Transition Areas for Infrastructure-Assisted Driving) project, funded by the Horizon 2020 EU Framework Programme for Research and Innovation.

\section{References}

Behrisch, M., Bieker, L., Erdmann, J., \& Krajzewicz, D. (2011). SUMO - Simulation of Urban MObility: An Overview. In Proceedings of SIMUL 2011, The Third International Conference on Advances in System Simulation. Barcelona: ThinkMind. Retrieved from http://www.thinkmind.org/index.php?view=instance \&instance=SIMUL+2011

Blythe, P., \& Curtis, A. (2004). Advanced driver assistance systems: Gimmick or reality? Presented at the 11th World Congress on ITS, Nagoya. Retrieved from https://www.researchgate.net/profile/Philip_Blythe/publication/233932657_Advanced_Driver_Assist ance_Systems_gimmick_or_reality/links/56b8843f08aebbde1a7f7907.pdf

Davis, L. C. (2004). Effect of adaptive cruise control systems on traffic flow. Physical Review E, 69(6). https://doi.org/10.1103/PhysRevE.69.066110

Hasebe, K., Nakayama, A., \& Sugiyama, Y. (2003). Dynamical model of a cooperative driving system for freeway traffic. Physical Review E, 68(2), 026102. https://doi.org/10.1103/PhysRevE.68.026102

Kesting, A., Treiber, M., \& Helbing, D. (2010). Enhanced intelligent driver model to access the impact of driving strategies on traffic capacity. Philosophical Transactions of the Royal Society of London A: Mathematical, Physical and Engineering Sciences, 368(1928), 4585-4605. https://doi.org/10.1098/rsta.2010.0084

Kesting, A., Treiber, M., Schönhof, M., \& Helbing, D. (2008). Adaptive cruise control design for active congestion avoidance. Transportation Research Part C: Emerging Technologies, 16(6), 668683. https://doi.org/10.1016/j.trc.2007.12.004

Krauß, S. (1998). Microscopic Modeling of Traffic Flow: Investigation of Collision Free Vehicle Dynamics (Doctoral Thesis). DLR-Forschungsbericht. Retrieved from http://elib.dlr.de/8380/

Liang, C.-Y., \& Peng, H. (1999). Optimal Adaptive Cruise Control with Guaranteed String Stability. Vehicle System Dynamics, 32(4-5), 313-330. https://doi.org/10.1076/vesd.32.4.313.2083

Liu, H., Kan, X., Wei, D., Chou, F.-C., Shladover, S. E., \& Lu, X.-Y. (2018). Using Cooperative Adaptive Cruise Control (CACC) to Form High-Performance Vehicle Streams - Microscopic Traffic Modeling (FHWA Exploratory Advanced Research Program No. Cooperative Agreement No. DTFH61-13-H-00013). University of California, Berkeley: California PATH Program.

Marsden, G., McDonald, M., \& Brackstone, M. (2001). Towards an understanding of adaptive cruise control. Transportation Research Part C: Emerging Technologies, 9(1), 33-51. https://doi.org/10.1016/S0968-090X(00)00022-X 
Milanés, V., \& Shladover, S. E. (2014). Modeling cooperative and autonomous adaptive cruise control dynamic responses using experimental data. Transportation Research Part C: Emerging Technologies, 48, 285-300. https://doi.org/10.1016/j.trc.2014.09.001

Milanés, V., \& Shladover, S. E. (2016). Handling Cut-In Vehicles in Strings of Cooperative Adaptive Cruise Control Vehicles. Journal of Intelligent Transportation Systems, 20(2), 178-191. https://doi.org/10.1080/15472450.2015.1016023

Milanes, V., Shladover, S. E., Spring, J., Nowakowski, C., Kawazoe, H., \& Nakamura, M. (2014). Cooperative Adaptive Cruise Control in Real Traffic Situations. IEEE Transactions on Intelligent Transportation Systems, 15(1), 296-305. https://doi.org/10.1109/TITS.2013.2278494

Naus, G. J. L., Vugts, R. P. A., Ploeg, J., van de Molengraft, M. J. G., \& Steinbuch, M. (2010). String-Stable CACC Design and Experimental Validation: A Frequency-Domain Approach. IEEE Transactions on Vehicular Technology, 59(9), 4268-4279. https://doi.org/10.1109/TVT.2010.2076320

Shladover, S., Su, D., \& Lu, X.-Y. (2012). Impacts of Cooperative Adaptive Cruise Control on Freeway Traffic Flow. Transportation Research Record: Journal of the Transportation Research Board, 2324, 63-70. https://doi.org/10.3141/2324-08

Treiber, M., \& Helbing, D. (2009). Microsimulations of Freeway Traffic Including Control Measures. At - Automatisierungstechnik Methoden Und Anwendungen Der Steuerungs-, RegelungsUnd Informationstechnik, 49(11/2001), 478. https://doi.org/10.1524/auto.2001.49.11.478

Treiber, M., Hennecke, A., \& Helbing, D. (2000). Congested traffic states in empirical observations and microscopic simulations. Physical Review E, 62(2), 1805-1824. https://doi.org/10.1103/PhysRevE.62.1805

Treiber, M., \& Kesting, A. (2013). Traffic Flow Dynamics: Data, Models and Simulation. Berlin Heidelberg: Springer-Verlag. Retrieved from //www.springer.com/us/book/9783642324598

van Arem, B., van Driel, C. J. G., \& Visser, R. (2006). The Impact of Cooperative Adaptive Cruise Control on Traffic-Flow Characteristics. IEEE Transactions on Intelligent Transportation Systems, 7(4), 429-436. https://doi.org/10.1109/TITS.2006.884615

Vander Werf, J., Shladover, S., Miller, M., \& Kourjanskaia, N. (2002). Effects of Adaptive Cruise Control Systems on Highway Traffic Flow Capacity. Transportation Research Record: Journal of the Transportation Research Board, 1800, 78-84. https://doi.org/10.3141/1800-10

VanderWerf, J., Shladover, S., Kourjanskaia, N., Miller, M., \& Krishnan, H. (2001). Modeling Effects of Driver Control Assistance Systems on Traffic. Transportation Research Record: Journal of the Transportation Research Board, 1748, 167-174. https://doi.org/10.3141/1748-21

Xiao, L., Wang, M., \& van Arem, B. (2017). Realistic Car-Following Models for Microscopic Simulation of Adaptive and Cooperative Adaptive Cruise Control Vehicles. Transportation Research Record: Journal of the Transportation Research Board, 2623, 1-9. https://doi.org/10.3141/2623-01 